\title{
Estudos longitudinais sobre o crescimento somático e desempenho motor: delineamentos, desafios, necessidades
}

\section{Longitudinal studies on somatic growth and motor performance: designs, challenges, and needs}

Simonete Pereira da Silva ${ }^{1}$

Gaston Beunen ${ }^{2}$

Duarte Luiz de Freitas ${ }^{3}$

José António Ribeiro Maia ${ }^{4}$

Resumo - O objetivo principal desta revisão é fornecer uma visão geral dos principais estudos longitudinais e longitudinais-mistos que se centraram sobre o crescimento somático, maturação biológica e, mais recentemente, também no desempenho físico. Somente foram considerados os estudos realizados na América do Norte, Europa e países de língua portuguesa. Em primeiro lugar, são apresentadas as principais considerações teóricas, características gerais, o delineamento do estudo e análise estatística multivariada dos dados. Na segunda parte, é edificado o panorama geral sobre os estudos emblemáticos de natureza longitudinal e longitudinal-mista. Finalmente, foram considerados alguns dos principais desafios que se colocam à pesquisa longitudinal.

Palavras-chave: Crescimento somático; Delineamentos longitudinais; Desempenho motor.

Abstract - The main objective of the present review of the literature was to provide an overview of the major longitudinal and mixed longitudinal studies focusing on somatic growth, biological maturation, and, more recently, physical performance. We selected studies conducted in the USA, Europe, and Portuguese speaking countries. First, the main theoretical considerations, general characteristics, study designs, statistical analyses, and methodological challenges of the data were presented. Next, we provided an overview of the most important longitudinal and mixed longitudinal studies. Finally, the major challenges of the ongoing studies were outlined.

Key words: Longitudinal designs; Motor performance; Somatic growth.

1 Universidade Regional do Cariri. Departamento de Educação Física. Crato, CE. Brasil

2 Katholieke Universiteit Leuven. Leuven. Bélgica

3 Universidade da Madeira. Departamento de Educação Física e Desporto. Região Autônoma da Madeira. Funchal. Portugal

4 Universidade do Porto. Faculdade de Desporto. Laboratório de Cineantropometria e Gabinete de Estatística. Porto. Portugal

Recebido em 21/05/12 Revisado em 14/06/12 Aprovado em 18/07/12 


\section{INTRODUÇÃO}

O primeiro estudo longitudinal, historicamente registrado, sobre o crescimento físico humano foi desenvolvido por Guineau de Montbeilard (1720-1780). A estatura do seu filho foi medida semestralmente desde o nascimento até aos 17 anos e 7 meses. Contudo, foi Franz Boas (1858 - 1942) quem deu o grande impulso à pesquisa Auxológica ao propor, formalmente, um delineamento sólido e métodos matemáticos para estudar aspectos das curvas da distância e da velocidade ${ }^{1}$. Ao introduzir as noções de velocidade e pico de crescimento, bem como ritmos diferenciados no crescimento, abriu a porta ao grande desafio interpretativo do significado da variabilidade do crescimento e desenvolvimento humanos.

Historicamente, é possível referenciar dois grandes períodos e localizações geográficas de pesquisa Autológica com enfoque primordial em estudos longitudinais. O primeiro, na América do Norte, entre 1930 e 1970. O segundo, na Europa, entre 1950 e 1980. O grande destaque deste "movimento" vai para investigadores renomados como Espenschade ${ }^{2}, \mathrm{Garn}^{3}$, Bayley ${ }^{4}$, Tanner ${ }^{5}$, Boyd ${ }^{6}$, Beunen et al., e Roche ${ }^{8}$. Todos conduziram pesquisas longitudinais com grandes dimensões amostrais que resultaram na produção da maior fatia da informação que possuímos atualmente sobre a descrição, interpretação, alcance clínico, educativo e social do crescimento e desempenho motor de crianças e jovens.

É inquestionável a riqueza dos estudos longitudinais por serem considerados, metaforicamente, uma espécie de relato da "história natural" do crescimento e desenvolvimento de diferentes atributos dos indivíduos que vão desde a concepção até ao final da vida ${ }^{1}$. A pesquisa longitudinal tem-se multiplicado nos últimos anos, tanto na extensão e variedade dos indicadores estudados, quanto na profundidade das suas abordagens. Contudo, nos países lusófonos e no domínio da Educação Física e Ciências do Esporte, tais delineamentos de pesquisas são ainda incipientes.

Este texto, um sumário brevíssimo, tem os seguintes objetivos: (1) enfatizar o valor e significado do alcance dos estudos longitudinais do crescimento e desempenho motor; (2) apresentar aspectos e desafios de tais delineamentos; (3) destacar algumas das principais linhas de pesquisas e, (4) apresentar as características de estudos desenvolvidos em diferentes países, salientando aspectos da sua estrutura organizacional.

A seleção dos estudos obedeceu a três critérios: (1) os delineamentos serem de natureza longitudinal; (2) o pioneirismo da pesquisa na época da sua implementação; (3) a circunstância de serem projetos emblemáticos em termos de representatividade, abrangência e impacto dos resultados. Finalmente, adicionamos alguns estudos realizados nos países lusófonos.

\section{DESENVOLVIMENTO}

\section{Direções da pesquisa}

Os mecanismos associados ao crescimento e desempenho motor possuem 
uma relação que inclui múltiplos fatores, designados genericamente de genéticos ${ }^{9}$, ambientais ${ }^{10}$ e culturais ${ }^{11,12}$. Atualmente, é bem conhecido que o crescimento físico é particularmente sensível às condições socioambientais (fenômeno designado por eco sensibilidade), dentre as quais destacam-se: a imigração, as doenças emergentes, os baixos níveis de atividade física, os diversos tipos de urbanização, disparidades em termos socioeconômicos e nos cuidados básicos de saúde, distintos hábitos nutricionais e poluição.

Atualmente, a ênfase da pesquisa longitudinal tem sido direcionada para cruzar olhares oriundos da Auxologia, Epidemiologia e Saúde Pública. O caráter interativo do crescimento, estilo de vida, saúde e tendência secular na maturação biológica, sobretudo, na precocidade da idade de menarca tem sido alvo de estudo em muitos países. A isto acresce, a construção de valores de referência para as populações de acordo com os seus contextos socioeconômicos, ambientais e culturais. Estas informações possibilitam uma intervenção em programas de Saúde Pública, Esporte infanto-juvenil e Educação Física.

A informação da base genética sobre o crescimento físico humano e maturação biológica, incluindo avaliações extensivas a famílias nucleares e gêmeos monozigóticos ou dizigóticos, tem sido utilizada para determinar a magnitude da importância dos fatores genéticos e a identificação dos genes responsáveis pela variabilidade interindividual e grupal ${ }^{9,13}$.

\section{Delineamentos}

O delineamento longitudinal puro (i.e. de grupo único) é considerado como a melhor estratégia para o estudo do crescimento e desempenho motor, no qual medidas dos mesmos sujeitos são realizadas repetidamente em intervalos pré-determinados ${ }^{14}$. Em função dos problemas associados ao delineamento puramente longitudinal, sobretudo, o tempo de recolha da informação, tem sido sugerido como alternativa o recurso a estudos com um delineamento longitudinal-misto, constituído por múltiplas coortes, com períodos de sobreposição e que possibilita a recolha de informação de forma mais célere. Uma vantagem do delineamento longitudinal-misto consiste na estimação da magnitude dos efeitos da idade, coorte e período de medição/avaliação na análise da mudança intraindividual e diferenças entre sujeitos ${ }^{15}$.

Numa perspectiva mais abrangente, tem sido introduzida uma nova estrutura na organização de pesquisa longitudinal com grande dimensão amostral que inclui a participação de diferentes países e/ou centros de pesquisa e que se denomina de delineamento multi-centro. Essa estrutura organizacional oferece múltiplas vantagens, de que destacamos: (1) a participação de diferentes países facilita a investigação de influências de origem étnica, geográfica, socioeconômica e cultural; (2) permite que informações de amostras de grande dimensão sejam obtidas num curto período de tempo; (3) a coleta de dados a partir de múltiplos centros conduz a uma maior heterogeneidade na amostra final, aumentando, assim, a gama e variabilidade interpretativa dos principais resultados. 
Independentemente da especificidade do delineamento, o sucesso de uma pesquisa longitudinal depende de integração sólida de três elementos indispensáveis: (1) a utilização de um modelo teórico para interpretar a mudança; (2) a implementação de um delineamento temporal que favoreça um quadro detalhado do fenômeno a estudar; (3) e um modelo estatístico que possibilite a análise multivariada do processo de mudança ${ }^{16}$.

\section{Desafios metodológicos}

A essência de um determinado delineamento longitudinal dirige o seu olhar para a descrição e interpretação da dinâmica do crescimento e desempenho motor, os padrões de "mudança" e estabilidade, bem como a magnitude das relações causais que procuram explicar o porquê da magnitude e sinal da mudança ${ }^{17}$. É evidente que tais delineamentos exigem uma organização complexa. Decorre, daqui, a necessidade de uma organização flexível, mas sólida na aquisição da informação a que se associa, necessariamente, a interpretação do quadro inter-relacional dos diferentes aspectos do crescimento e desenvolvimento motor. Num estudo longitudinal puro, é exigido um tempo relativamente longo para a recolha completa da informação. Os desafios a enfrentar num projeto desta natureza são elevados, de que destacamos: (1) financiamento; (2) defasamento temporal entre a recolha de dados e publicação da informação; (3) dimensão amostral e respectivo abandono; (4) manutenção da equipe de campo; (5) controle e qualidade da informação e efeitos de aprendizagem associado à retestagem; (6) organização e manutenção da base de dados; (7) desenvolvimento de estratégias de motivação para manutenção da amostra; e (8) uso de procedimentos estatísticos complexos para responder às diferentes questões de pesquisa ${ }^{14,17}$.

\section{Análise da informação}

Da combinação dos propósitos do estudo e da metodologia associada à implementação de pesquisa de natureza longitudinal e/ou longitudinal-mista, emerge uma grande massa de dados que causa sérias dores de cabeça aos pesquisadores. Atualmente, estão disponíveis não somente modelos estatísticos com elevada flexibilidade, elegância e robustez, mas também algoritmos eficazes que se encontram implementados em softwares de fácil acesso e utilização.

Algumas das avenidas de análise situam-se nos seguintes planos: (1) $\mathrm{Na}$ construção de cartas percentílicas. Atualmente, dispõe-se de vários modelos, sendo que dois deles são os mais divulgados: o de Cole e Green ${ }^{18}$ designado de LMS e o modelo de Rigby e Stasinopoulos ${ }^{19}$; (2) A interpretação adequada da mudança intraindividual e das diferenças interindividuais são problemas complexos se adicionarmos a presença de variáveis mediadoras e de preditores da mudança que são invariantes e/ou se alteram com o tempo do estudo. Neste domínio complexo, a modelação hierárquica multinível tem produzido um esforço notável na construção de um modo de pensar e analisar a informação e (3) Identificação de grupos de risco, clusters de sujeitos cujas trajetórias temporais são distintas ${ }^{20}$. 


\section{ESTUDOS LONGITUDINAIS - EXEMPLOS ILUSTRATIVOS}

Desde o início do século passado, começaram a ser implementadas nos centros de investigação da América do Norte e Europa várias pesquisas longitudinais de larga escala com enfoque no crescimento e desempenho motor. Para localizar os estudos longitudinais produzidos, foram realizadas pesquisas nas bases de dados Pubmed, Scopus, Sportdiscus e Scielo, utilizando-se como termos de indexação (em inglês e português): longitudinal, crescimento somático, maturação biológica e desempenho motor.

Nesta parte do texto, destaca-se, muito brevemente, algumas das principais pesquisas desenvolvidas na América do Norte, Europa e em países lusófonos. O Quadro 1 refere-se aos estudos provenientes da América do Norte.

Quadro 1. Principais estudos longitudinais desenvolvidos na América do Norte.

\begin{tabular}{|c|c|c|c|}
\hline Estudo & Localização & Amostra, duração & Indicadores estudados \\
\hline $\begin{array}{l}\text { Child Welfare Re- } \\
\text { search Station' }\end{array}$ & $\begin{array}{l}\text { lowa e Chicago } \\
\text { EUA } \\
1914\end{array}$ & $\begin{array}{l}1924 \text { Crianças } \\
\text { (861 đđ } 1063 \text { } \text { ) } \\
\text { Idade: } 3 \text { Anos } \\
\text { Duração: } 9 \text { anos }\end{array}$ & Altura, peso e capacidade vital. \\
\hline $\begin{array}{l}\text { Harvard Growth } \\
\text { Study' }\end{array}$ & $\begin{array}{l}\text { Medford, Revere e } \\
\text { Beverly - Boston } \\
\text { EUA, } 1922\end{array}$ & $\begin{array}{l}3600 \text { Crianças de ambos } \\
\text { os sexos } \\
\text { Idades } \approx 5 \text { e } 6 \text { Anos } \\
\text { Duração: } 12 \text { anos }\end{array}$ & $\begin{array}{l}\text { Medidas somáticas; Maturação biológica; } \\
\text { Testes de desempenho escolar. }\end{array}$ \\
\hline $\begin{array}{l}\text { Child Research } \\
\text { Council Study }{ }^{1}\end{array}$ & $\begin{array}{l}\text { Denver, Colorado } \\
\text { EUA } \\
1930\end{array}$ & $\begin{array}{l}334 \text { Crianças = Sendo } 256 \\
\text { indivíduos acompanhados } \\
\text { do nascimento até à idade } \\
\text { adulta. } \\
\text { Duração: } 36 \text { anos }\end{array}$ & $\begin{array}{l}\text { Medidas somáticas; Composição corporal; } \\
\text { Maturação biológica; Variáveis hemato- } \\
\text { lógicas; Tamanho do coração; Aspectos } \\
\text { nutricionais; Concentração de proteínas; } \\
\text { Pressão arterial; eletrocardiograma; Tole- } \\
\text { rância ao exercício; Taxa metabólica basal; } \\
\text { Testes psicológicos. }\end{array}$ \\
\hline $\begin{array}{l}\text { Fels Longitudinal } \\
\text { Study }^{1}\end{array}$ & $\begin{array}{l}\text { Yellow Springs, Ohio } \\
\text { EUA } \\
1929\end{array}$ & $\begin{array}{l}1036 \text { Crianças, das quais } \\
537 \text { foram avaliadas du- } \\
\text { rante } 8 \text { anos; } \\
\text { Cerca de } 20 \text { crianças foram } \\
\text { acompanhadas desde a } \\
\text { gestação até à vida adulta. } \\
\text { Em curso }\end{array}$ & $\begin{array}{l}\text { Medidas somáticas; Maturação biológica; } \\
\text { Composição corporal; Aptidão física; Ativi- } \\
\text { dade física; Fatores de risco para doenças } \\
\text { crônicas; Marcadores genéticos; Movimen- } \\
\text { tos fetais; Aspectos nutricionais; Capaci- } \\
\text { dade auditiva; Comportamento familiar; } \\
\text { Desempenho escolar. }\end{array}$ \\
\hline $\begin{array}{l}\text { Medford Boys } \\
\text { Growth Study }{ }^{21}\end{array}$ & $\begin{array}{l}\text { Oregon, } \\
\text { EUA } \\
1956\end{array}$ & $\begin{array}{l}\approx 400 \precsim \\
7 \text { a } 18 \text { anos } \\
12 \text { anos }\end{array}$ & $\begin{array}{l}\text { Medidas somáticas; Maturação biológica; } \\
\text { Somatótipo; Força; Desempenho motor; } \\
\text { Estado nutricional; Desempenho escolar; } \\
\text { Testes psicológicos e de interesse voca- } \\
\text { cional. }\end{array}$ \\
\hline $\begin{array}{l}\text { Saskatchewan } \\
\text { Child Growth and } \\
\text { Development } \\
\text { Study }\end{array}$ & $\begin{array}{l}\text { Saskatoon, } \\
\text { Canadá } \\
1964\end{array}$ & $\begin{array}{l}207 \text { ô } \\
98 \text { }+ \\
\text { Idade: } 7 \text { anos } \\
\text { Duração: } 10 \text { anos }\end{array}$ & $\begin{array}{l}\text { Medidas somáticas; Composição corporal; } \\
\text { Maturação biológica; Capacidade vital; Ati- } \\
\text { vidade física; Aptidão física geral e especí- } \\
\text { fica; VO } \mathrm{V}_{2} \text { max; Performance motora; Hábitos } \\
\text { e estilos de vida; Traços da personalidade; } \\
\text { Avaliação sociológica e comportamental; } \\
\text { Estatuto sócioeconômico. }\end{array}$ \\
\hline
\end{tabular}


O primeiro grande estudo longitudinal sobre o crescimento físico humano desenvolvido na América do Norte teve início em 1914, no Child Welfare Research Station da Universidade de Iowa ${ }^{1}$ e foi conduzido por um grupo de psicólogos e fisiologistas liderados por Bird T. Baldwin. Um dos principais propósitos foi a construção de cartas de crescimento.

O segundo grande estudo longitudinal americano foi o do Harvard Growth Study ${ }^{1}$ conduzido por W.F. Dearborn. Esta pesquisa apresenta uma amostra bastante expressiva para um estudo longitudinal, cerca de 3600 crianças. Iniciou em 1922 em Medford. Apesar do inevitável drop out amostral, ao final de 12 anos de pesquisa, cerca de 1000 crianças ainda estavam sendo acompanhadas. Uma das grandes preocupações deste estudo foi o rigor nas avaliações das medidas antropométricas. Dearborn, responsável pelo treinamento da equipe de campo composta por 26 pessoas, estabeleceu estreitos limites de tolerância para cada medida e a sua réplica, por forma a garantir a precisão das medidas efetuadas. Os resultados desta pesquisa forneceram um notável material de análise sobre o crescimento na adolescência que mais tarde foi publicado por Frank Shuttleworth (1899 - 1958).

Em 1923, o Centro Médico da Universidade do Colorado iniciou The Child Research Council Study ${ }^{1}$, uma pesquisa de caráter epidemiológico desenvolvida com o intuito de detectar doenças pediátricas associadas a desvios de normalidade do crescimento. Este foi, sem dúvida, um estudo emblemático em termos de pioneirismo nas variáveis estudadas. O caráter multidisciplinar marcou uma nova fase de pesquisa, na época, ao introduzir, para além das características somáticas, aspectos fisiológicos, nutricionais e psicológicos. Os períodos de medições foram mais frequentes do que os outros estudos longitudinais: avaliações mensais até um ano de idade, depois desta fase, a cada três meses até o salto pubertário e finalmente, a cada ano, até atingir a maturidade. Com base no modelo matemático de Gompertz (modelo matemático para uma série temporal, onde o crescimento está mais lento no início e no final de um determinado período de tempo), foi possível a construção de curvas individuais de velocidade da altura de 24 meninos e 24 meninas.

O Fels Longitudinal Study ${ }^{1}$ é considerado o maior e o mais longo estudo de natureza longitudinal jamais desenvolvido nos Estados Unidos. O seu início foi em 1929, por iniciativa de Lester Sontag, com apoio da Fels Foundation. Pretendeu-se responder a uma questão básica: por que é que as pessoas são diferentes ao longo da sua trajetória de vida? Com o passar dos anos, foram incorporadas outras questões relacionadas com estilos de vida, síndrome metabólica e genética.

O Medford Boys Longitudinal Study foi desenvolvido através da Universidade de Oregon, sob a direção de Harrison Clark ${ }^{21}$. Teve início em 1956 e foi concluído em 1968. Este estudo apresentou um delineamento simultaneamente longitudinal e transversal. É considerado um dos primeiros estudos que enfatizaram a performance esportiva. Os objetivos centrais foram a construção de curvas de velocidade e distância para as variáveis 
somáticas e motoras, bem como relacionar os aspectos físicos, maturacionais, psicológicos, nutricionais e de interesse pelo esporte.

No Canadá, um dos estudos mais emblemáticos foi desenvolvido por Donald Bayley entre 1964 e 1973, envolvendo um grupo de 207 meninos e 98 meninas de 7 anos que foram avaliados, anualmente, até os 15 - 16 anos. O Saskatchewan Child Growth and Development Study ${ }^{22}$ foi uma pesquisa marcada, sobretudo, pela extensão e profundidade dos indicadores estudados. Dentre os objetivos destacam-se: a construção de curvas de crescimento e velocidade numa variedade de características físicas e motoras; análise do padrão de correlações existentes num conjunto de indicadores de maturação biológica, desempenho motor, hábitos e estilos de vidas sobre o crescimento físico humano.

$\mathrm{Na}$ Europa, os estudos longitudinais tiveram sua origem após a II Guerra Mundial. Embora muita pesquisa tenha sido conduzida no âmbito da Psicologia Comportamental, a sua orientação principal foi no campo da Medicina. No Quadro 2, é apresentado um resumo das características de alguns dos principais estudos longitudinais e longitudinais-mistos desenvolvidos em diferentes países da Europa.

Quadro 2. Principais estudos longitudinais sobre o crescimento desenvolvidos na Europa.

\begin{tabular}{|c|c|c|c|}
\hline Estudo & Localização & Amostra, duração & Indicadores estudados \\
\hline $\begin{array}{l}\text { Harpender Gro- } \\
\text { wth Study }{ }^{1,5,23,24}\end{array}$ & $\begin{array}{l}\text { Harpenden } \\
\text { Inglaterra } \\
1948\end{array}$ & $\begin{array}{l}450 \AA \\
269 \stackrel{+}{\uparrow} \\
\text { A partir dos } 3 \text { anos. }\end{array}$ & $\begin{array}{l}\text { Medidas somáticas; Composição corporal; } \\
\text { Somatótipo; Maturação biológica; Avalia- } \\
\text { ções ortodônticas. }\end{array}$ \\
\hline $\begin{array}{l}\text { First Zurich } \\
\text { Longitudinal } \\
\text { Study }^{25,26}\end{array}$ & $\begin{array}{l}\text { Zurich - Suíça } \\
\text { 1a Fase: } 1954 \text { - } 1978 \\
\text { 2a Fase: } \\
\text { 1974 - } 1998 \\
\text { 3a Fase: desde } \\
\text { 1999 }\end{array}$ & 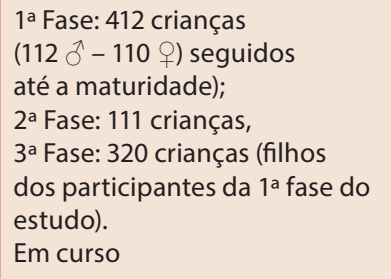 & $\begin{array}{l}\text { Medidas somáticas; Composição corporal; } \\
\text { Maturação biológica. }\end{array}$ \\
\hline $\begin{array}{l}\text { Leuven Longitu- } \\
\text { dinal Study on } \\
\text { Lifestyle, Fitness } \\
\text { and Health } \\
\text { an,28 }\end{array}$ & $\begin{array}{l}\text { Bélgica (amostra nacional) } \\
\text { 1a Fase: } \\
\text { 1969- } 1974 \\
\text { 2a Fase: } \\
\text { 1979- } 1980 \\
\text { 3a Fase: } \\
\text { 1986 - } 2004\end{array}$ & $\begin{array}{l}\text { 1a Fase: } \\
\text { Idades } 12-20 \text { anos } \\
\text { 2a Fase: } 588 \\
\text { 3a Fase: } 174 \\
\text { Atualmente } 90 \text { sujeitos com } 35 \\
\text { a } 40 \text { anos são acompanhados. } \\
\text { Em curso. }\end{array}$ & $\begin{array}{l}\text { Medidas somáticas; Composição corporal; } \\
\text { Somatótipo; Maturação biológica; Aptidão } \\
\text { física; Variáveis fisiológicas e socioculturais; } \\
\text { Fatores genéticos; Fatores de risco de doen- } \\
\text { ças cardiovasculares. }\end{array}$ \\
\hline $\begin{array}{l}\text { Amsterdam } \\
\text { Growth Study }{ }^{29}\end{array}$ & $\begin{array}{l}\text { Amsterdam - Holanda } \\
\text { 1a Fase: } \\
\text { 1977 - } 1980 \\
\text { 2 } \text { aase: }^{\text {1980 - } 1955} \\
\text { 3a Fase: } \\
\text { 1985 - } 1991\end{array}$ & 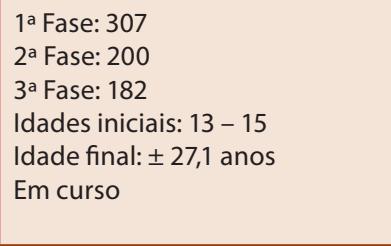 & $\begin{array}{l}\text { Medidas somáticas; Maturação biológica; } \\
\text { Aptidão física; Atividade física; Fatores de } \\
\text { risco de doenças cardiovasculares; Estilo de } \\
\text { vida; Densidade óssea; Avaliação postural; } \\
\text { Parâmetros fisiológicos; Hábitos nutricio- } \\
\text { nais; Parâmetros psicossociais; Traços da } \\
\text { personalidade; Saúde mental. }\end{array}$ \\
\hline
\end{tabular}

O Harpender Growth Study foi idealizado por E. R. Bransby, um nutricionista do Ministério da Saúde inglês britânico, mas foram James Tanner e Reginald Whitehouse $\mathrm{e}^{1,23,24}$ os pesquisadores responsáveis pelo estudo que teve início em 1948 e terminou em 1971. Os principais interesses situaram- 
-se no campo da endocrinologia, nutrição e auxologia. Com o intuito de aumentar a precisão e manuseio dos equipamentos tradicionais, foram elaboradas e estandardizadas técnicas de medições e construídos seus instrumentos antropométricos. A partir deste estudo, foram padronizadas algumas técnicas antropométricas constantes do Programa Biológico Internacional, bem como a popularização da avaliação dos caracteres sexuais secundários descritos por Tanner ${ }^{1}$. A frequência de avaliações durante o período pubertário foi uma grande vantagem deste estudo em relação aos anteriores, o que proporcionou uma adequada eficiência no alinhamento dos dados para a construção de curvas da velocidade para as diferentes medidas antropométricas. A grande massa de dados deste estudo nunca foi editada na íntegra; no entanto, a partir de 1966, foram publicadas inestimáveis contribuições que marcaram o panorama atual da auxologia, das quais destacamos, as cartas de distância e de velocidade para a altura e o peso corporal de crianças Britânicas, desde o nascimento até os 18 anos.

O First Zurich Longitudinal Study $y^{25,26}$ foi realizado em Zurique, tendo sido iniciado em 1954, com uma amostra de 412 crianças, das quais 222 foram acompanhadas do nascimento até atingir a maturidade. Inicialmente, o investigador responsável foi Andrea Prader (1919 - 2001), um pediatra interessado em pesquisar problemas endócrinos associados ao crescimento normal e anormal. Para além das variáveis somáticas, de composição corporal e maturação esquelética, avaliou um indicador que nenhuma outra pesquisa havia estudado anteriormente, o volume testicular, enquanto índice biológico dos caracteres sexuais secundários, de enorme importância em Pediatria. Para o efeito, foi desenvolvido o orquidômetro, designado de Prader, que mais adiante passou a ser amplamente adotado em clínica pediátrica. Andrea Prader empenhou-se na investigação de como as crianças tendem a aumentar a sua velocidade de crescimento, para além do esperado, se por qualquer circunstância foi desviada do seu canal de crescimento. Este fenômeno foi designado de catch up growth ${ }^{27}$, sendo hoje um termo universalmente adotado pelos pesquisadores da área.

O Leuven Longitudinal Study on Lifestyle, Fitness and Health teve origem em dois estudos anteriormente existentes: o Leuven Growth Study of Belgian Boys (1969 - 1974) e o Leuven Growth Study of Flemish Girls $(1979-1980)^{28}$. Foi desenvolvido através do Instituto de Educação Física da Universidade Católica de Leuven e foi conduzido por M. Ostyn e J. Simons, aos quais se associou, alguns anos depois, Gaston Beunen. O estudo apresenta um delineamento longitudinal-misto com uma abordagem multifacetada. Na primeira fase, avaliou cerca de 21.175 rapazes, durante 6 anos. Na segunda fase, acompanhou longitudinalmente 588 rapazes, oriundos da amostra inicial. Na terceira fase, retomou a pesquisa quando estes tinham 30, 35 e 40 anos de idade, respectivamente. Atualmente, o estudo continua com cerca de 90 indivíduos (e suas famílias) da amostra inicial, sendo avaliados anualmente.

O Amsterdam Growth Study teve início em 1977 e está sediado na Universidade de Amsterdam, concretamente nos Departamentos 
de Ciências da Saúde e Psicopatologia. Os Professores Han Kemper, Verschuur e mais tarde Willem Van Mechelen ${ }^{29}$ conduziram o projeto, cujo maior destaque vai não só para a multiplicidade e profundidade de indicadores pesquisados, mas também para a sua inovação ao introduzir a avaliação da densidade óssea, características posturais, aspectos psicossociais e saúde mental. A amostra foi constituída por crianças oriundas de duas escolas secundárias da região de Amsterdam, que foram seguidas dos 13 aos 27 anos. Diversas publicações têm sido produzidas ao longo desta pesquisa, proporcionando um vasto quadro de informações sobre o crescimento e desenvolvimento numa perspectiva de análise ao longo da vida.

No espaço da lusofonia, apenas Portugal e Brasil desenvolveram pesquisas temporais com enfoque no crescimento físico e desempenho motor. O Quadro 3 apresenta as características dos estudos desenvolvidos em Portugal.

Quadro 3. Estudos longitudinais desenvolvidos em Portugal.

\begin{tabular}{|c|c|c|c|}
\hline Estudo & Localização & Amostra, duração & Indicadores estudados \\
\hline $\begin{array}{l}\text { Estudo de Crescimento da } \\
\text { Madeira }^{30}\end{array}$ & $\begin{array}{l}\text { Região Autônoma da } \\
\text { Madeira - Portugal } \\
2002\end{array}$ & 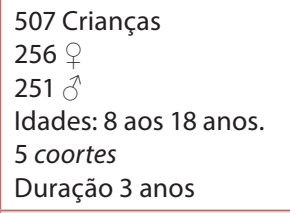 & $\begin{array}{l}\text { Medidas somáticas; Maturação bioló- } \\
\text { gica; Aptidão física; Atividade física; } \\
\text { Características socioeconômicas. }\end{array}$ \\
\hline $\begin{array}{l}\text { O Estudo da Região Autônoma } \\
\text { do Açores }{ }^{31}\end{array}$ & $\begin{array}{l}\text { Região Autônoma } \\
\text { dos Açores - Portugal } \\
2003\end{array}$ & $\begin{array}{l}1159 \text { Crianças } \\
6-19 \text { Anos } \\
4 \text { coortes } \approx 250 \text { sujeitos } \\
\text { por coorte } \\
\text { Duração } 4-5 \text { anos }\end{array}$ & $\begin{array}{l}\text { Medidas somáticas; Coordenação mo- } \\
\text { tora, Maturação biológica, Composição } \\
\text { corporal; Coordenação motora; Ativi- } \\
\text { dade física; Motivação para a prática } \\
\text { esportiva; Síndrome metabólica. }\end{array}$ \\
\hline
\end{tabular}

O primeiro estudo longitudinal desenvolvido em Portugal foi conduzido por Freitas et al. ${ }^{30}$ intitulado, O Estudo de Crescimento da Madeira. Partindo de uma proposta multidisciplinar, o estudo apresentou um delineamento longitudinal-misto, com 5 coortes estudadas por três anos consecutivos. Uma das principais novidades do estudo foi construir cartas percentílicas para os diferentes indicadores do crescimento físico humano, maturação esquelética e aptidão física da população escolar da Região Autônoma da Madeira.

O segundo estudo também com abordagem multidisciplinar, foi conduzido por Maia e colaboradores ${ }^{31}$ na Região Autônoma dos Açores. Essencialmente, e estudo objetivou descrever e interpretar as mudanças e implicações pedagógicas e epidemiológicas que ocorrem no crescimento somático, desenvolvimento motor, hábitos de atividade física e fatores de risco de doenças cardiovasculares. A amostra foi constituída por 4 coortes de 250 sujeitos cada, que foram seguidos durante $4-5$ anos. As novidades da pesquisa residem na avaliação da motivação para a prática esportiva e no estudo da síndrome metabólica de famílias nucleares de crianças e jovens normoponderais, com sobrepeso e obesos. 
No Quadro 4, estão apresentados os estudos longitudinais e longitudinais-mistos sobre o crescimento e desempenho motor desenvolvidos no Brasil, até o presente momento.

Quadro 4. Estudos longitudinais desenvolvidos no Brasil.

\begin{tabular}{|c|c|c|c|}
\hline Estudo & Localização & Amostra, duração & Indicadores estudados \\
\hline Projeto Ilha Bela ${ }^{32}$ & $\begin{array}{l}\text { Ilha Bela, São Paulo } \\
1978\end{array}$ & $\begin{array}{l}62 \text { } \\
\text { Idades: } 10 \text { a } 15 \text { Anos } \\
\text { Duração: } 5 \text { anos }\end{array}$ & $\begin{array}{l}\text { Medidas somáticas; Aptidão física; } \\
\text { Maturação sexual }\end{array}$ \\
\hline $\begin{array}{l}\text { Crescimento e aptidão } \\
\text { física relacionada à } \\
\text { saúde em escolares }^{33}\end{array}$ & $\begin{array}{l}\text { Santa Catarina } \\
1992\end{array}$ & $\begin{array}{l}84 \text { Crianças } \\
\text { Idades: } 7 \text { - } 10 \text { anos } \\
\text { Duração: } 4 \text { anos }\end{array}$ & $\begin{array}{l}\text { Altura e peso } \\
\text { Aptidão física }\end{array}$ \\
\hline $\begin{array}{l}\text { Estudo das caracterís- } \\
\text { ticas antropométricas } \\
\text { de escolares }^{34}\end{array}$ & $\begin{array}{l}\text { Santa Catarina } \\
2000\end{array}$ & $\begin{array}{l}298 \text { Crianças } \\
161 \hat{\jmath} \\
137 \stackrel{+}{ } \\
\text { Duração: } 4 \text { anos }\end{array}$ & Altura e peso \\
\hline PROESP - Brasil 35 & $\begin{array}{l}\text { Canoas } \\
\text { Rio Grande do Sul } \\
2008\end{array}$ & $\begin{array}{l}70 \text { Crianças } \\
35 \hat{\jmath} \\
35 q \\
\text { Duração } 4 \text { anos }\end{array}$ & $\begin{array}{l}\text { Altura, peso, dobras de adiposidade } \\
\text { subcutâneas; Aptidão física }\end{array}$ \\
\hline $\begin{array}{l}\text { Estudo do crescimento } \\
\text { e desenvolvimento } \\
\text { motor de escolares de } \\
\text { Muzambinho }\end{array}$ & $\begin{array}{l}\text { Muzambinho - Minas } \\
\text { Gerais } \\
2007\end{array}$ & $\begin{array}{l}\text { 1a Fase }=471 \text { crianças de ambos } \\
\text { os sexos } \\
7 \text { - } 14 \text { Anos } \\
\text { Duração: } 2,5 \text { anos } \\
\text { 2a Fase }=163 \text { crianças } \\
\text { Idades: } 6 \text { a } 10 \text { anos } \\
\text { 200 Famílias nucleares } \\
\text { Duração: } 4-5 \text { anos }\end{array}$ & $\begin{array}{l}\text { Medidas somáticas; Aptidão física; } \\
\text { Coordenação motora; Atividade } \\
\text { física; Avaliação postural; Estatuto } \\
\text { socioeconômico; Variáveis psicoló- } \\
\text { gicas; Fatores de risco cardiovascular } \\
\text { dos pais e das crianças. }\end{array}$ \\
\hline $\begin{array}{l}\text { Projeto Crescer com } \\
\text { Saúde no Cariri }{ }^{37-39}\end{array}$ & $\begin{array}{l}\text { Região do Cariri Ceará } \\
\text { (Juazeiro do Norte, } \\
\text { Crato e Barbalha). } \\
2010\end{array}$ & $\begin{array}{l}\text { Delineamento longitudinal-misto } \\
\text { Amostra }=436 \\
\text { Delineamento Transversal } \\
\text { Amostra }=4005 \\
\text { Idades } 7 \text { a } 17 \text { anos } \\
\text { Ambos os sexos } \\
\text { Duração: } 3 \text { anos }\end{array}$ & $\begin{array}{l}\text { Medidas somáticas; Aptidão física; } \\
\text { Maturação esquelética e sexual; } \\
\text { Atividade física habitual; } \mathrm{VO}_{2} \text { max e } \\
\text { estatuto socioeconômico. }\end{array}$ \\
\hline
\end{tabular}

Uma das primeiras iniciativas de se estudar o crescimento físico humano e desempenho motor, ao longo do tempo, no Brasil, foi efetuada no âmbito do Projeto Ilhabela. Trata-se de um estudo longitudinal misto realizado desde 1978, em Ilhabela, litoral norte de São Paulo. Com base nos dados de 62 meninas participantes deste projeto, Biassio et al. ${ }^{32}$ publicaram um estudo sobre o impacto da menarca nas variáveis antropométricas e neuromotoras de adolescentes de baixo nível socioeconômico, avaliadas durante 5 anos. Nahas et al. ${ }^{33}$ com um delineamento de grupo único, investigaram, anualmente, a estatura, peso e aptidão física relacionada a saúde de 84 crianças e jovens de 10 a 14 anos. Também Waltrick e Duarte ${ }^{34}$ conduziram uma pesquisa a partir de um delineamento simultaneamente longitudinal e transversal, somente da estatura e do peso, que foram medidos, anualmente, durante 4 anos. No âmbito do PROESP Brasil (Projeto Esporte Brasil), alguns estudos descritivos em torno das questões relacionadas à estrutura somática e desempenho motor foram publicadas por Bergmann et al ${ }^{35}$.

O Estudo de Muzambinho ${ }^{36}$, em curso, é um exemplo de uma nova fase de pesquisas sobre o crescimento físico humano e envolve investigadores 
do Brasil, Portugal e Moçambique. Com base numa abordagem multidisciplinar acerca do crescimento físico humano e desenvolvimento motor de crianças e jovens, o estudo é estruturado em duas fases: a primeira teve um duplo propósito: (1) investigar aspectos diversificados do crescimento físico humano e (2) quantificar a associação entre a coordenação motora e a aptidão física. A segunda centrou-se na pesquisa da agregação familiar das capacidades motoras e dos fatores de risco cardiovascular.

O Projeto Crescer com Saúde no Cariri ${ }^{37-39}$ é a mais recente pesquisa concluída nesta área temática. $\mathrm{O}$ estudo foi desenvolvido na região do Cariri Cearense, com delineamento simultaneamente longitudinal-misto e transversal. O objetivo central deste estudo foi descrever e interpretar o quadro dinâmico e multifacetado de interações que se estabelecem entre crescimento somático, maturação biológica, aptidão física, atividade física habitual e estatuto socioeconômico de crianças e jovens da região do Cariri Cearense. Participaram desta pesquisa, crianças e adolescentes oriundos de escolas públicas e privadas de Juazeiro do Norte, Crato e Barbalha, de ambos os sexos e idades compreendidas entre os 7 e os 17 anos. Dos resultados deste estudo destacam-se a construção de cartas de referências para o peso, altura, índice de massa corporal, bem como para uma vasta gama de testes motores para crianças e adolescentes de ambos os sexos. As principais conclusões deste estudo apontaram para o impacto de vários fatores, incluindo genéticos, socioeconômicos, geográficos e culturais e muito provavelmente, a interação entre esses indicadores a modularem o crescimento e o desempenho motor das crianças e adolescentes caririenses.

\section{CONSIDERAÇÕES FINAIS}

Não obstante o esforço empreendido em fornecer informações relevantes no domínio interativo da Auxologia e das Ciências do Esporte, importa salientar algumas limitações deste breve texto. Contudo, é importante reconhecer que as inovações metodológicas e de análise estatística apresentadas nos diferentes estudos, qualquer que seja a sua proveniência geográfica, coloca alguns entraves a uma comparação dos seus principais resultados e conclusões. É evidente que este artigo possui várias limitações, sendo que a mais importante refere-se às escolhas/opções dos seus autores. Assim, salienta-se os seus limites: (1) nos estudos selecionados privilegiarem, sobretudo, pesquisas com delineamento longitudinal e longitudinal-misto, enfatizando o seu pioneirismo na época e local da sua implementação; (2) serem investigações cuja dimensão amostral é relativamente elevada para delineamentos longitudinais; (3) a consideração da sua abrangência face ao recurso a múltiplos indicadores em diferentes domínios, e (4) o impacto acadêmico e social dos resultados.

De um modo geral, verifica-se que os estudos norte americanos e europeus foram estruturados com base numa abordagem fortemente multidisciplinar e, ao mesmo tempo, iam atualizando novas questões e introduzindo instrumentação mais inovadora e moderna. Nos países 
lusófonos, a estrutura dos delineamentos e a abrangência dos propósitos não é tão vasta, contudo, não deixa de ser importante pelo seu legado histórico, pelos desafios dos seus resultados e pela abertura que oferece a novos pesquisadores do território desenvolvimentista quer se situem no plano Auxológico, Epidemiológico ou na área das Ciências do Esporte.

O delineamento multi-centro é umas das estratégias mais promissoras para a investigação nos países que compõem o território lusófono, através da colaboração interinstitucional entre os seus pesquisadores. Tal iniciativa permitirá uma maior abrangência na abordagem dos problemas a que se adicionará uma forte componente transcultural que importa interpretar à luz de um pensamento Antropológico e Social renovado por uma leitura fortemente ecológica.

Alguns dos principais desafios que se colocam aos pesquisadores situam-se no campo da utilização de modernas tecnologias e padronização dos métodos de recolha da informação para permitirem uma maior capacidade de associação com os atuais problemas de pesquisas. Do mesmo modo, a utilização de sofisticadas técnicas de análise estatística e modelos matemáticos permitirá um maior alcance na análise e interpretação inovadora dos dados.

\section{REFERÊNCIAS BIBLIOGRÁFICAS}

1. Tanner JM. A History of the Study of the Human Growth: Cambridge University Press; 1981.

2. Espenschade A. Motor Performance in Adolescence. Including the Study of Relationships With Measures of Physical Growth and Maturity. Washington, DC: Society for Research in Child Development; 1940.

3. Garn SM. Relative Fat Patterning: An Individual Characteristic. Hum Biol 1955;27:75-89.

4. Bayley N. Growth Curves of Height and Weigth by Age for Boys and Girls, Scaled According to Physical Maturity. J Pediatr 1956;48:187-94.

5. Tanner JM. Growth at Adolescence. 2a ed. Oxford: Blackwell Scientific; 1962.

6. Boyd Edit. Origins of the Study of Human Growth. Colorado, EUA. 1980.

7. Beunen G, Malina R, Van't Hof MA, Simons J, Ostyn M, Renson R, Van Gerven D. Adolescent Growth and Motor Performance. A Longitudinal Study in Belgian Boys. Illinois: Human Kinetics; 1988.

8. Roche AF. Growth, Maturation and Body Composition. The Fels Longitudinal Study 1921 - 1929: Cambridge University Press; 1992.

9. Bouchard C, Malina RM, Pérusse L. Genetics of Fitness and Physical Performance. United States: Human Kinetics; 1997.

10. Martorell R, Mendoza F, Castillo R. Poverty and Stature in Children. In: Waterlow JC, editor. Linear Retardation in Less Developed Countries. New York: Raven Press; 1988. p. 57-70.

11. Allen LH. Nutritional Influences on Linear Growth: A General Review. Eur J Clin Nutr 1994;(48):75-89.

12. Frisancho AR. Developmental Adaptation to High Altitude Hipoxia. Int J Biometerol 1977;21:135-46.

13. Onis M, Garza C, Victora CG, Onyango AW, Frongillo EA, Martines J. The WHO Multicentre Growth Reference Study: Planning, Study Design, and Methodology. Food Nutr Bull 2004;25(1):S15-S26.

14. Menard S. Longitudinal Research. London: Sage University Paper; 2002. 
15. Van Mechelen W, Mellenberg, GJ. Problems and Solutions in Longitudinal Research: From Theory to Practice. Int J Sports Med 1997;18:S238-S45.

16. Prahl-Andersen B, Kowalski CJ. Analysis of Cohort Effects in Mixed Longitudinal Data Sets. Int J Sports Med 1997;18:S186-S94.

17. Collins LM. Analysis of Longitudinal Data: The Integration of Theoretical Model, Temporal Design, and Statistical Model. Annu Rev Psychol 2006;57:505-28.

18. Cole TJ, Green PJ. Smoothing Reference Centile Curves: The LMS Method and Penalized Likelihood. Statistics in Medicine 1992;11:1306-19.

19. Rigby RA, Stasinopoulos DM. Smooth Centile Curves for Skew and Kurtosis Data Modelled Using the Box-Cox Power Exponential Distribution. Stat Medic 2004;23(19):3053-76.

20. Himes JH. Why Study Child Growth and Maturation? In: Hauspie RC, Cameron N, Molinari L. (eds). Methods in Human Growth Research. United Kingdom: Cambridge University Press; 2004:3-26.

21. Clark HH. Contributions and implications of the Meldford Boy's Growth Study. Oregon University; 1968.

22. Mirwald RL. Saskatchewan Growth and Development Study. In: Ostyn M, Beunen G, Simons J, editors. International Series on Sports Sciences: Kinantropometry II. Baltimore: University Park Press;1980:289-05.

23. Tanner JM, Whitehouse R, Takaishi M. Standards from Birth to Maturity for Height, Weight, Height Velocity, and Weight Velocity: British Children, 1965, Part II. Arch Dis Child 1966;41:454-71.

24. Tanner JM, Whitehouse R, Takaishi M. Standards from Birth to Maturity for Height, Weight, Height Velocity, and Weight Velocity: British Children, 1965, Part II. Arch Dis Child 1966;41:613-35.

25. Largo RH, Prader A. Pubertal development in Swiss boys. Helv Paediatr Acta 1983;38(3):211-28.

26. Prader A, Largo RH, Molinari L, Issler C. Physical growth of Swiss children from birth to 20 years of age. First Zurich longitudinal study of growth and development. Helv Paediatr Acta 1989;52 (Suppl 1),S1-125.

27. Prader A. Catch-up Growth. Postgrad Med J 1978;54(1):133-46.

28. Matton L, Duvigneaud N, Wijndaele K, Philippaerts R, Duquet W, Beunen G, at al. Secular Trends in Anthropometric Characteristics, Physical Fitness, Physical Activity, and Biological Maturation in Flemish Adolescents Between 1969 and 2005. Am J Hum Biol 2007:19(3):345-57.

29. Twisk J, Kemper H. Design of the Amsterdam Growth Study. In: Kemper HC, editors. The Amsterdam Growth Study: A Longitudinal Analysis of Health, Fitness and Lifestyle. Amsterdam: Human Kinectics; 1995:6-16.

30. Freitas D, Maia JAR, Beunen G, Lefevre JA, Claessens AL, Marques, et al. Crescimento Somático, Maturação Biológica, Aptidão Física, Actividade Física e Estatuto Socioeconómico de Crianças e Adolescentes Madeirenses - O Estudo de Crescimento da Madeira. SAEFD Universidade da Madeira. Portugal; 2002.

31. Maia JAR, Lopes VL. Um Olhar Sobre Crianças e Jovens da Região Autónoma dos Açores. Implicações para Educação Física, Desportos e Saúde. Porto: FCDEF; 2003.

32. Biassio LG, Matsudo SM, Matsudo VK. Impacto da Menarca nas Variáveis Antropométricas e Neuromotoras da Aptidão Física, Analisado Longitudinalmente. Rev Bras Cienc Mov 2004;12(2):97-101.

33. Nahas MV, Petroski EL, Jesus JF, Silva OJ. Crescimento e Aptidão física Relacionada à Saúde em Escolares de 7 a 10 anos - Um Estudo Longitudinal. Rev Bras Cienc Esporte 1992;14(1):7-17.

34. Waltrick A, Duarte MF. Estudo das Características Antropométricas de Escolares de 7-17 Anos. Uma Abordagem Longitudinal-Mista e Transversal. Rev Bras Cineantropom Desempenho Hum 2000;2(1):17-30.

35. Bergmann G, Bergmann ML, Pinheiro, ES, Moreira RB, Marques AC, Gaya AC. Estudo Longitudinal do Crescimento Corporal de Escolares de 10 a 14 anos: Dimorfismo Sexual e Pico de Velocidade. Rev Bras Cineantropom Desempenho Hum 2008;10(3):249-54. 
36. Basso L, Meira Júnior C, Oliveira JA, Forjaz CL, Souza JA, Prista A, at el. Crescimento e desenvolvimento motor de escolares de Muzambinho: Um Estudo com Implicações Acadêmicas, Sociais e de Política Interinstitucional. Rev Port Cien Desp 2009;9(2-3):247-57.

37. Silva S, Maia JAR, Claessens AL, Beunen G, Huiqi P. Growth references for Brazilian children and adolescents: Healthy growth in Cariri study. Ann Hum Biol 2012;(39)1:11-8.

38. Silva S, Beunen G, Maia JAR. Valores normativos do desempenho motor de crianças e adolescentes: O estudo longitudinal-misto do Cariri. Rev bras Educ Fís Esporte 2011;25(1):111-25.

39. Silva S, Freitas D, Beunen G, Maia JAR. Maturação Biológica: da sua relevância à aprendizagem do Método TW3. Rev Bras Cineantropom Desempenho Hum 2010;12(5):352-8.

\section{Endereço para correspondência}

Simonete Pereira da Silva

Universidade Regional do Cariri Departamento de Educação Física Rua Cel. António Luiz, 1161. Pimenta CEP: 63.100-000 - Crato. CE

E-mail: simonete_silva@yahoo.com.br 